\title{
ANTIMICROBIAL RESISTANCE AMONG STREPTOCOCCUS AGALACTIA COLONIZERSIN PREGNANT WOMEN
}

\author{
Sherin Ahmed Sami El Masry*, Noha Alaa El-Din Fahim*, and \\ Mona Byoumee Ragay**
}

\begin{abstract}
*Clinical Pathology Department, Faculty of Medicine, Ain Shams University and **El galaa teaching hospital, Cairo, Egypt

Corresponding author

Mona Bayoumee Ragay;

Mobile: (+2) 01002972958

E.mail:

Drmonmonbayoumee@yahoo.com

Received: $3 / 5 / 2021$

Accepted: 27/5/2021
\end{abstract}

Online ISSN: 2735-3540

\section{ABSTRACT:}

Background: Streptococcus agalactiae (Group B Streptococcus, $G B S)$, a leading cause of sepsis and meningitis in infants, can be transmitted vertically from mother to infant during passage through the birth canal. Detection of GBS colonization in perinatal women is a major strategy for the prevention of postpartum neonatal disease.

Aim of the work: To determine the in vitro antimicrobial susceptibility profile of group $\beta$ Streptococcus in cases of pregnant females between 34 and 37 weeks of gestation for guiding the antimicrobial prophylaxis for cases of GBS colonized pregnant females.

Patients and methods: This study included 29 isolates of group $\beta$ Streptococcus recovered from rectovaginal swabs taken from 112 pregnant women between 34 and 37 weeks of gestation. All swabs were inoculated on blood agar plate with bacitracin disc. Isolates that give B-haemolysis on blood agar and are bacitracin resistant were identified by catalase and CAMP test as group B-streptococci and they were 29 isolates. Antibiotic susceptibility by disc diffusion was done to the 29 isolates, using cation adjusted Mueller Hinton agar using the following antibiotic discs: penicillin, vancomycin, clindamycin, erythromycin, levofloxacin and cefotaxime according to the Clinical and Laboratory Standard Institute .

Results: The highest level of resistance was reported against cefotaxime where 20/29 (69\%) were resistant. Whilst the minimum resistance was exhibited against levofloxacin with a $27.5 \%(8 / 29)$ resistance rate.

Conclusion: The prevalence rate of GBS colonization among pregnant women included in the study was 29/112 (25.9\%) which is concordant with many other local and international studies. Thus, it is very important to expand the prenatal GBS screening among pregnant women to avoid the maternal and neonatal complications. It is recommended to perform antimicrobial susceptibility to pregnant women with GBS.

Keywords: GBS, antimicrobial resistance, rectovaginal swabs.

\section{INTRODUCTION:}

Group B Streptococcus (GBS) is a human commensal, where, the gastrointestinal tract being the natural reservoir and more likely the source for vaginal colonization. At any given time, 10-
$40 \%$ of healthy adults are commonly colonized by GBS in the gastrointestinal and genital tract but remain asymptomatic. Vaginal colonization is unusual in childhood but becomes more common in late adolescence $^{[1]}$. 
Group $\quad \beta$ Streptococcus (GBS) or Streptococcus agalactiae has been considered one of the most important risks for the development of neonatal diseases. GBS is often associated with medical intercurrences during pregnancy and the postpartum period and can be associated with life-threatening disease in newborns due to sepsis, pneumonia, and meningitis ${ }^{[2]}$.

Among pregnant women GBS carriage rate in the vaginal and rectal microbiota ranges from $10 \%$ to $37 \%$ and is similar in both developing and developed countries. Large variations in colonization rates can be observed and can relate to ethnicity, body sites sampled, microbiological procedures performed and population studied ${ }^{[1]}$.

Prenatal GBS screening is recommended by the Centers for Disease Control and Prevention (CDC) by means of specimens harvestedrom the vaginal in troitus and perianal region from all the pregnant women between 35 and 37weeks of gestation $^{[1]}$.

Intrapartum antibiotic prophylaxis (IAP) reduce both the vertical transmission of Streptococcus agalactiae or group $\beta$ Streptococcus (GBS) and the early onset of neonatal sepsis. However, existing guidelines do not recommend that antimicrobial susceptibility testing (AST) be routinely performed. Penicillin or ampicillin are indicated as first-choice antibiotics, cefazolin being an alternative in the case of history of mild allergic reactions, and vancomycin orclindamycin an alternative in severe reactions. Concerns about IAP pertain potential toxicity and, mainly, potential pressure towards antibiotic resistance among GBS strains ${ }^{[1]}$.
Penicillin remains the agent of choice for intrapartum antibiotic prophylaxis, with ampicillin as an acceptable alternative. Penicillin-allergic women who have a history of anaphylaxis, angioedema, respiratory distress or urticaria following administration of penicillin should receive cefazolin $^{[3]}$.

The high rate of resistance in GBS strongly supports the current Centers for Disease Control and Prevention recommenddation that antibiotic susceptibility testing be performed if erythromycin or clindamycin therapy is needed to prevent neonatal GBS infection $^{[4]}$.

\section{AIM OF The WORK:}

To determine the in vitro antimicrobial susceptibility profile of group $\beta$ Streptococcus in cases of pregnant females between 34 and 37 weeks of gestation for guiding the antimicrobial prophylaxis for cases of GBS colonized pregnant females.

\section{PATIENTS AND METHODS:}

This study included 29isolates of group $\beta$ Streptococcus recovered from rectovaginal swabs taken from 112 pregnant women between 34 and 37 weeks of gestation. This study was performed during the period between December 2017 and May 2018. All swabs were inoculated on blood agar plate and were confirmed to be GBS by conventional species identification techniques including catalase test, bacitracin disc. CAMP test was done for B haemolytic and bacitracin resistant isolates (29 isolates) (Diagrams 1, 2). 


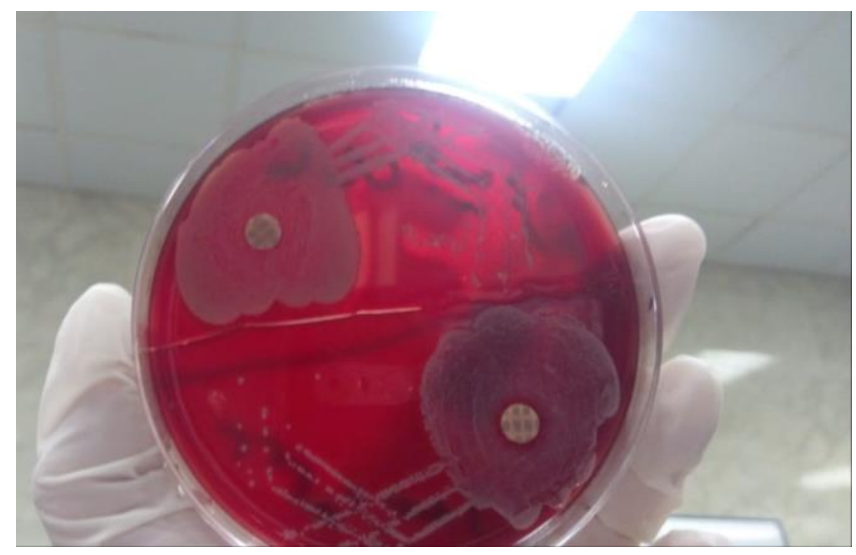

Diagram (1): The beta hemolytic colonies of GBS on blood agar resistant to Bacitracin

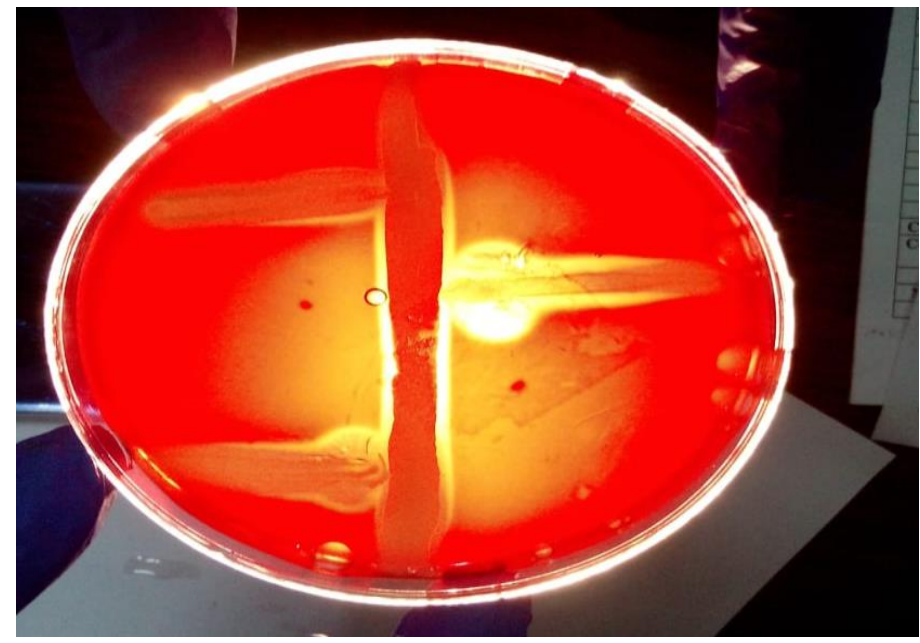

Diagram (2):The enhanced beta hemolytic colonies of GBS on blood agar indicating positive CAMP test

Antibiotic susceptibility by disc $\operatorname{disc}^{[5]}$. diffusion was being done to all isolates using cation adjusted Mueller Hinton agar using the following antibiotic discs: penicillin, vancomycin, clindamycin, erythromycin, levofloxacin and cefotaxime. All used antibiotic discs were purchased from (Oxoid, UK).

\section{Procedure:}

Discs containing cefotaxime, clindamycin, erythromycin, levofloxacin, penicillin and vancomycin were placed on Muller Hinton blood agar plate where 0.5 McFarland bacterial suspension was inoculated. The plates were incubated overnight at $37^{\circ} \mathrm{C}$.Sensitivity of the bacteria to one of the antibiotics was measured by the zone of inhibition around the antibiotic

\section{Interpretation:}

Interpretation was performed according to the Clinical and Laboratory Standard Institute ${ }^{[6]}$.

Statistical Analysis: The collected data were revised, coded, tabulated and introduced to a PC using Statistical Package for Social Science (SPSS 20). Data were presented and suitable analysis was done according to the type of data obtained for each parameter.

\section{RESULTS:}

Table (1) shows the percentage range of parity between $0.9 \%$ and $35.7 \%$, the 
percentage range of abortions between $3.1 \%$ and $43.8 \%$ and the percentage range of gravida between $1.1 \%$ and $29.5 \%$ among pregnant females included in the study.

Table (1): The percentage range of parity, abortions and gravid among pregnant females included in the study

\begin{tabular}{|c|c|c|c|c|c|c|}
\hline & \multicolumn{2}{|c|}{ Parity } & \multicolumn{2}{|c|}{ Abortions } & \multicolumn{2}{|c|}{ Gravida } \\
\hline & $\mathbf{N}$ & $\%$ & $\mathbf{N}$ & $\%$ & $\mathbf{N}$ & $\%$ \\
\hline PG & 24 & $21.4 \%$ & & & & \\
\hline 1 & 40 & $35.7 \%$ & 14 & $43.8 \%$ & 1 & $1.1 \%$ \\
\hline 2 & 33 & $29.5 \%$ & 10 & $31.3 \%$ & 26 & $29.5 \%$ \\
\hline 3 & 14 & $12.5 \%$ & 7 & $21.9 \%$ & 25 & $28.4 \%$ \\
\hline 4 & 1 & $.9 \%$ & 0 & $0.0 \%$ & 19 & $21.6 \%$ \\
\hline 5 & 0 & $0.0 \%$ & 1 & $3.1 \%$ & 9 & $10.2 \%$ \\
\hline 6 & 0 & $0.0 \%$ & 0 & $0.0 \%$ & 6 & $6.8 \%$ \\
\hline 7 & 0 & $0.0 \%$ & 0 & $0.0 \%$ & 2 & $2.3 \%$ \\
\hline
\end{tabular}

Table (2) shows that there was no statistically significant difference between mean of both age and gestational age (GA) GBS and non-GBS groups as regards the

Table (2): The age and Gestational age (GA) mean in GBS and non-GBS groups.

\begin{tabular}{|c|c|c|c|c|c|c|c|}
\hline \multirow{2}{*}{} & \multicolumn{4}{|c|}{ Blood agar } & \multicolumn{4}{c|}{ t test } \\
\cline { 2 - 8 } & \multicolumn{2}{|c|}{ Non- GBS } & \multicolumn{2}{c|}{ GBS } & t & p value & sig. \\
\cline { 2 - 8 } & Mean & SD & Mean & SD & -0.80 & 0.429 & NS \\
\hline Age & 27.61 & 5.43 & 28.55 & 5.79 & -0.76 & 0.447 & NS \\
\hline G.A. & 35.39 & 1.45 & 35.58 & 1.06 & \\
\hline
\end{tabular}

The results of pus cells and Gram stain diagnostic or confirmatory method of GBS could not be correlated with growth on colonization in our study (Table2) (Diagram blood agar. So, they can neither be used as 3).

Table (3): Results of blood agar inoculation, bacitracin, CAMP test, pus cells and Gram stain of the specimens.

\begin{tabular}{|l|c|c|l|}
\hline \multicolumn{2}{|c|}{} & $\mathrm{N}$ & \multicolumn{1}{c|}{$\%$} \\
\hline \multirow{3}{*}{ Blood agar } & NO haemolysis & 79 & $70.5 \%$ \\
\cline { 2 - 4 } & Bhaemolysis & 33 & $29.5 \%$ \\
\hline \multirow{3}{*}{ CAMP test } & Negative & 0 & $0 \%$ \\
\hline \multirow{3}{*}{ Bacitracin } & Positive & 29 & $100 \%$ \\
\hline & Sensitive & 83 & $74.1 \%$ \\
\hline \multirow{3}{*}{ Gramscells } & Resistant & 29 & $25.9 \%$ \\
\cline { 2 - 4 } & Negative & 102 & $91.1 \%$ \\
\cline { 2 - 4 } & Positive & 10 & $8.9 \%$ \\
\cline { 2 - 4 } & gram-ve bacilli & 23 & $8.0 \%$ \\
\cline { 2 - 4 } & gram+ve cocci & 80 & $71.4 \%$ \\
\hline
\end{tabular}




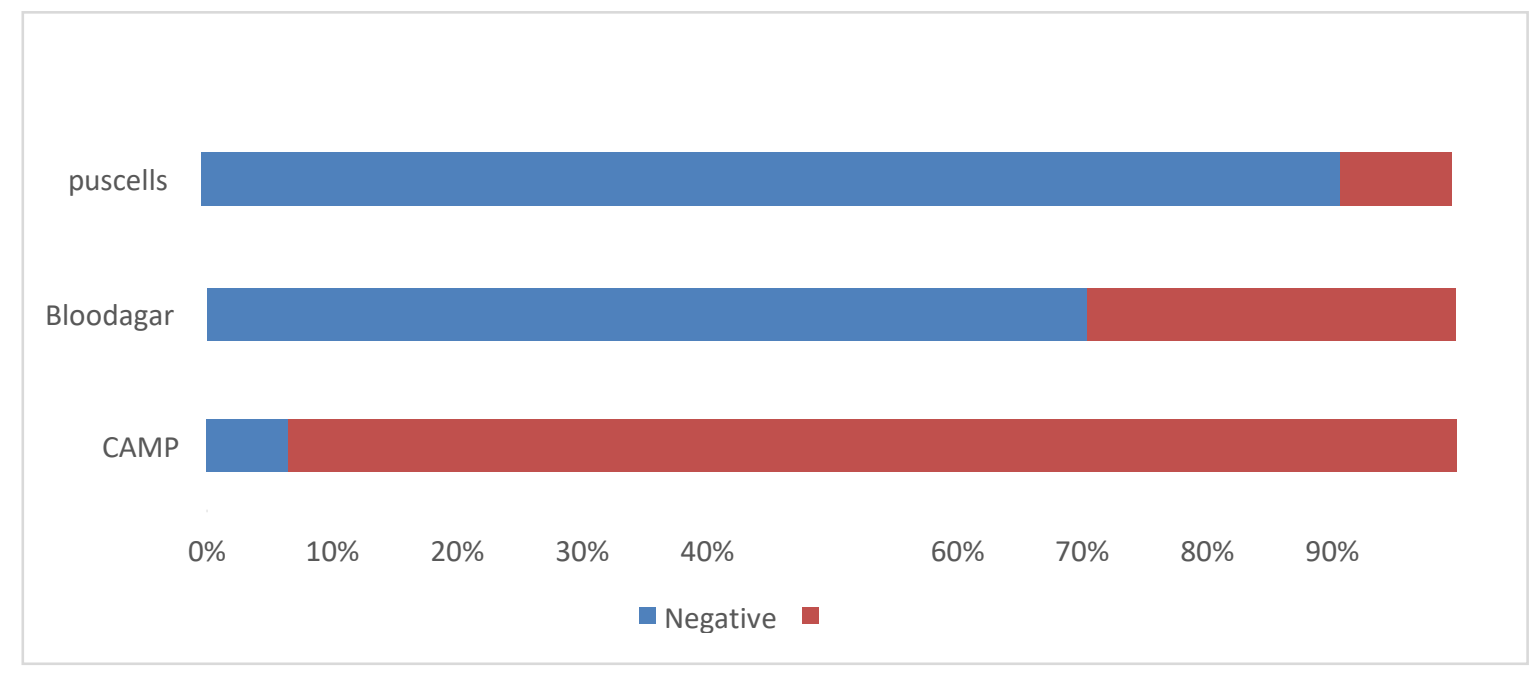

Diagram (3): Results of blood agar, pus cells and CAMP test

Regarding the results of antimicrobial the minimum resistance was exhibited susceptibility testing, the highest level of resistance was reported against cefotaxime where 20/29 (69\%) were resistant. Whilst against levofloxacin with a $27.5 \%(8 / 29)$ resistance rate. The results are summarized in table (4), diagrams ( 4 \& 5).

Table (4): Results of antibiotic susceptibility of GBS isolates by disc diffusion method

\begin{tabular}{|l|c|c|c|c|}
\hline \multirow{2}{*}{} & \multicolumn{2}{|c|}{ Resistant } & \multicolumn{2}{c|}{ Sensitive } \\
\cline { 2 - 5 } & $\mathrm{N}$ & $\%$ & $\mathrm{~N}$ & $\%$ \\
\hline Levofloxacin & 8 & $27.50 \%$ & 21 & $72.50 \%$ \\
\hline Penicillin & 11 & $37.90 \%$ & 18 & $62.10 \%$ \\
\hline Vancomycin & 13 & $44.80 \%$ & 16 & $55.20 \%$ \\
\hline Cefotaxime & 20 & $69 \%$ & 9 & $31 \%$ \\
\hline Clindamycin & 15 & $51.70 \%$ & 14 & $48.30 \%$ \\
\hline Erythromycin & 11 & $37.90 \%$ & 18 & $62.10 \%$ \\
\hline
\end{tabular}

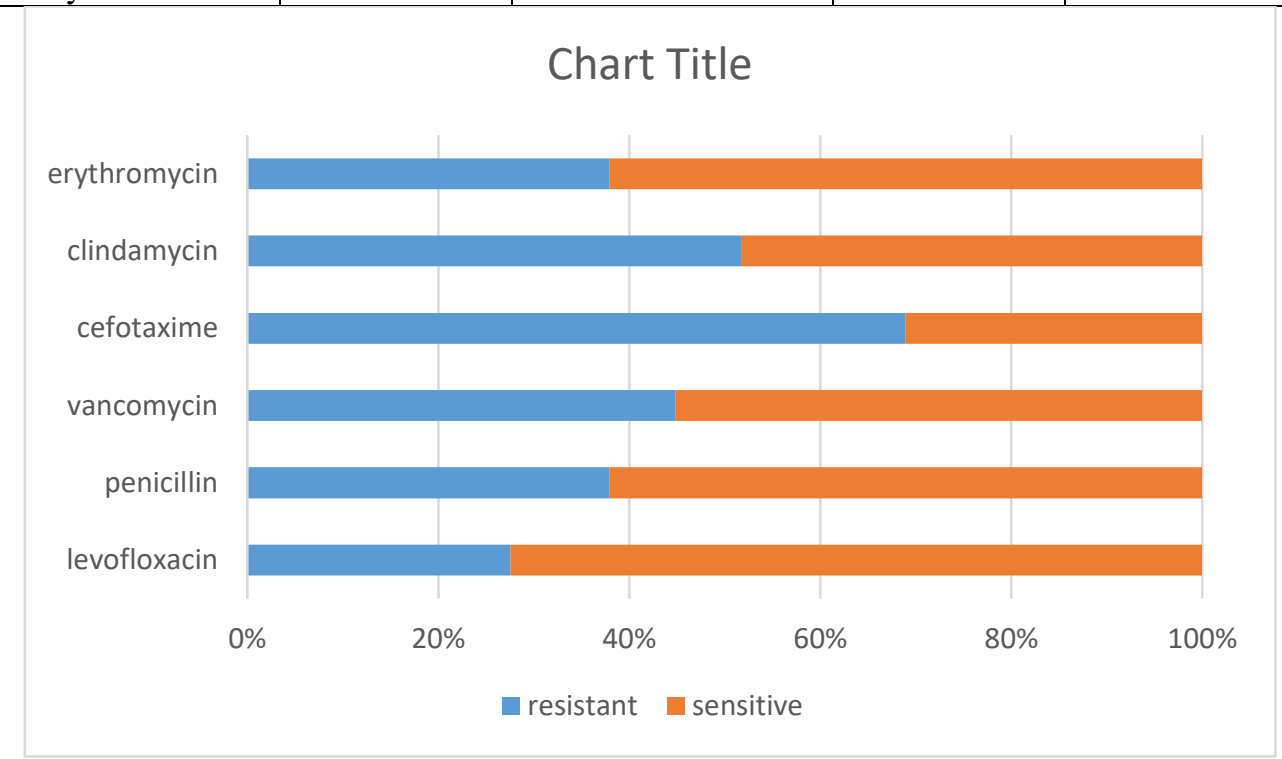

Diagram (4): Results of antibiotic susceptibility of GBS isolates by disc diffusion method. 


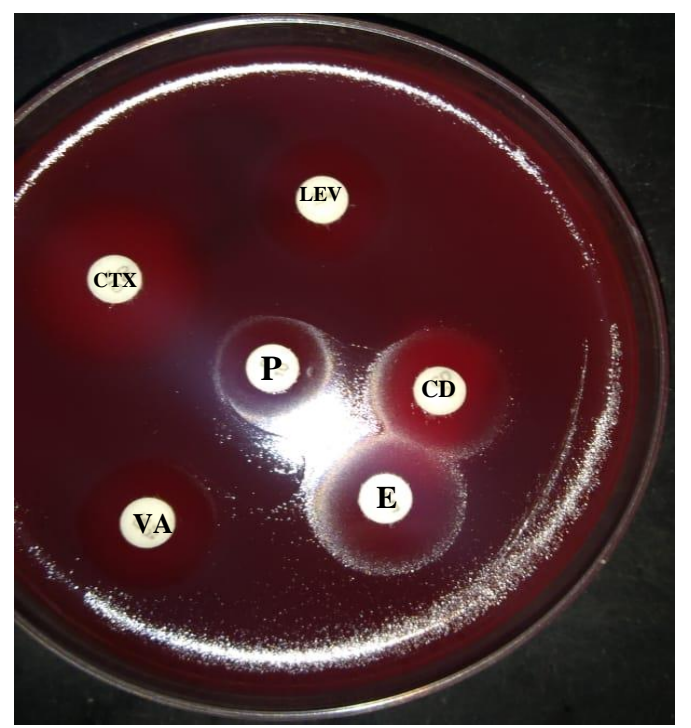

Diagram (5): Antibiotic susceptibility testing of GBS by disc diffusion showing resistance to penicillin, clindamycin, levofloxacin, erythromycin and sensitive to cefotaxime

\section{DISCUSSION:}

Streptococcus agalactiae (Group B Streptococcus, GBS), a leading cause of sepsis and meningitis in infants, can be transmitted vertically from mother to infant during passage through the birth canal. Detection of GBS colonization in perinatal women is a major strategy for the prevention of postpartum neonatal disease. The U.S. Centers for Disease Control and Prevention recommends that all women under govaginal-rectal screening for GBS colonization at 35-37 weeks of gestation ${ }^{[7]}$.

In the present study, a prevalence rate of $25.9 \%$ was found for GBS among pregnant females. These results were concordant with other studies carried out in Egypt [8\&9]. Similarly, another study performed by Sadaka et al. ${ }^{[10]}$ at Alexandria reported a similar prevalence rate.

Other countries also showed agreement with the prevalence rate of GBS found in our study. Da Rocha et al. ${ }^{[11]}$ from Brazil, and Morita et al. ${ }^{[7]}$ from Japan reported prevalence rates of $(28.2 \%$, and $22.4 \%)$ respectively.

On the other hand, other studies in different countries showed disagreement with the prevalence rate found in our study as a study carried out in Jordan showed lower prevalence rate of $19.5 \%{ }^{[12]}$.

Regarding the results of antimicrobial susceptibility testing, the highest level of resistance was reported against Cefotaxime where 20/29 (69\%) were resistant. Whilst the minimum resistance was exhibited against levofloxacin with a $27.5 \%(8 / 29)$ resistance rate.

In this study, although levofloxacin is the drug with the highest sensitivity, however, it is not the drug of choice as it, unfortunately, has a high risk of spontaneous abortion [13], thus it is only used in lifethreatening conditions.

Our study results were concordant in a great scale with other studies as Mengist et al. $^{[14]}$ with results of penicillin resistance of $77.3 \%$, and erythromycin resistance of $22.6 \%$.

However, another study by Jalalifar and other ${ }^{[15]}$ in (2019) showed similar results to ours regarding clindamycin resistance $(47 \%)$ and erythromycin resistance $(52 \%)$ while their isolates displayed much lower resistance to penicillin $(8 \%)$ and cefotaxime $(8 \%)$. 


\section{Conclusion:}

The prevalence rate of GBS colonization among pregnant women included in the study was $25.9 \%$ (29/112). Thus, it is very important to expand the prenatal GBS screening among pregnant women to avoid the maternal and neonatal complications.

It is recommended to perform antimicrobial susceptibility to pregnant women with GBS.

\section{REFERENCES:}

1. Verani J, McGee L, Schrag S (2010): Prevention of perinatal group Bstreptococcal disease: revised guidelines from CDC. MMWR RecommRep.59:1-36.

2. Hamedi A, Akhlaghi $F$, Seyedi $S$ et al., (2012): Evaluation of group B Streptococci colonization ratein pregnant women and their newborn. Acta MedIran; 50:805-8.

3. Committee on Infectious Diseases and Committee on Fetus and Newborn (2010): Recommendations for the prevention of perinatal group B streptococcal (GBS) disease.

4. Heelan JS, Hasenbein ME, McAdam AJ (2004): Resistance of group B streptococcus to selected antibiotics, including erythromycin and clindamycin. Journal of Clinical Microbiology; 42(3):1263-4.

5. Jonasson E, Matuschek E, Kahlmeter G (2020): The EUCAST rapid disc diffusion method for antimicrobial susceptibility teasting directly from positive blood culture bottles. Journal of Antimicrobial Chemotherapy, Volume 75, Issue 4, April, Pages 968-978

6. Clinical and Laboratory Standards Institute (2015). Performance standards for antimicrobial susceptibility testing. Twenty-fifth informational supplement M100-S25. Wayne, PA, USA: Clinical and Laboratory Standards Institute.

7. Morita T, Feng D, Kamio Y, Kanno I, Somaya T, Imai K, Inoue M, Fujiwara M,
Miyauchi A (2014): Evaluation of chrom ID strepto Basascreen in gmedia for Streptococcus agalactiae. BMC infectious diseases; 14(1):1-4.

8. Tash RM, Elsaid GT, Mohamed IA. (2019): Carriage of Streptococcus agalactiae among Pregnant Women in an Egyptian University Hospital, Serotypes Distribution and Antibiotics Susceptibility. Department of Medical Microbiology \& Immunology, Zagazig Faculty of Medicine, Zagazig, Egypt. Department of Obstetrics and Gynecology, Benha Faculty of Medicine, Benha University, Benha, Egypt Egyptian Journal of Medical MicrobiologyVolume28 /No.3,79-84:

9. El Shahaway AA, El Maghraby HM, Mohammed HA, Abd Elhady RR, Abdelrhman AA(2019): Diagnostic performanceofdirectlatexagglutination,postenrichment latex agglutination and culture methods in screening of group B streptococci in late pregnancy: a comparative study. Infection and drug resistance;12:2583.

10. DaRochaJZ, FeltracoJ, RadinV, GonçalvesCV, daSilvaPE, VonGrollA (2020): Streptococcus agalactiaecolonizationandscreeningapproac hinhigh-

riskpregnantwomeninsouthernBrazil.TheJour nalofInfectioninDevelopingCountries;14(04) :332-40.

11. SadakaSM,AlyHA,MeheissenMA,OriefYI, $\operatorname{ArafaBM}(2018)$ :GroupBstreptococcalcarria ge,antimicrobialsusceptibility,andvirulencer elatedgenesamongpregnantwomeninAlexan dria,Egypt.AlexandriaJournalofMedicine;54 (1):69-76.

12. ClouseK,ShehabiA,SuleimatAM,FaouriS,Kh uri-

BulosN,AlJammalA,ChappellJ,FortnerKB,C hambyAB,RandisTM,RatnerAJ(2019):High prevalence of Group B Streptococcus colonization among pregnant women in Amman, Jordan. BMC pregnancy and childbirth;19(1):1-8.

13. Muanda FT, Sheehy O, Bérard A (2017): Use of antibiotics during pregnancy and risk of spontaneous abortion. Cmaj; 189(17):E625-33. 


\section{Sherin Ahmed Sami El Masry, et al.}

14. Mengist HM, Zewdie O, Belew A, Dabsu R (2017): Prevalence and drug susceptibility pattern of group B Streptococci (GBS) among pregnant women attending antenatal care (ANC) in Nekemte Referral Hospital (NRH), Nekemte, Ethiopia. BMC research notes; 10(1):1-6.

15. Jalalifar S, Havaei SA, Motallebirad T,
Moghim S, Fazeli H, Esfahani BN (2019): Determination of surface proteins profile, capsular genotyping, and antibiotic susceptibility patterns of Group B Streptococcus isolated from urinary tract infection of Iranian patients. BMC research notes; 12(1):1-6.

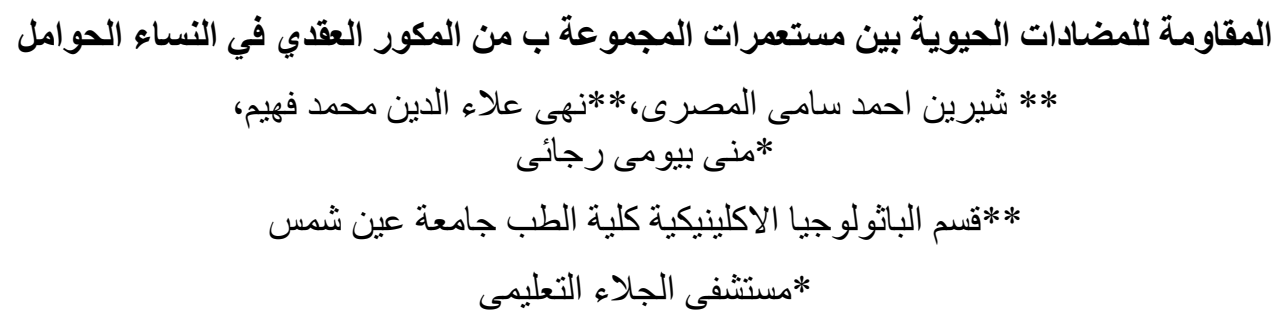

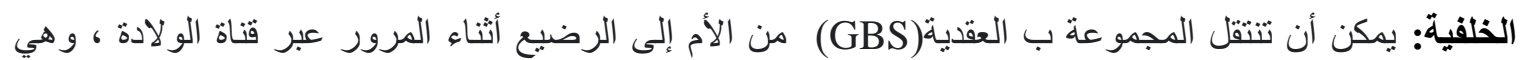

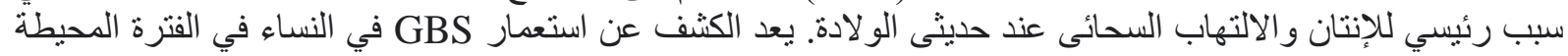
بالو لادة استر اتيجية رئيسية للوقاية من امر اض حديثي الو لادة.

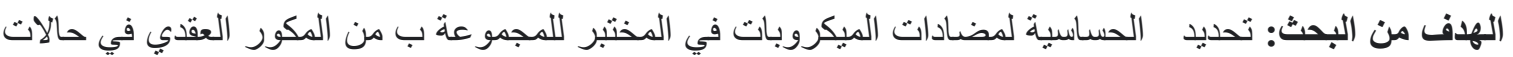

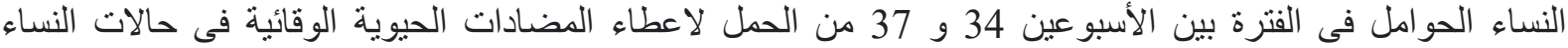
الحو امل المصابات بالمجمو عة ب من المكور العقدى . الاعن

المرضى و طرق البحث : اشتملت هذه الدر اسة على 29 فصيلة من المجموعة ب من من المكور العقدى تم تجميعها من

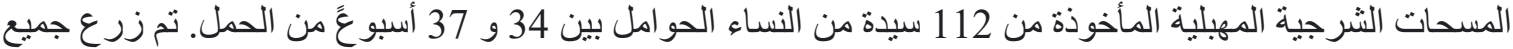

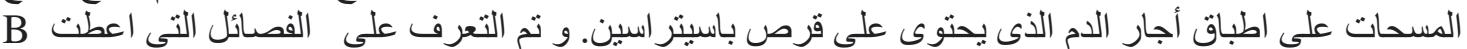
haemolysis

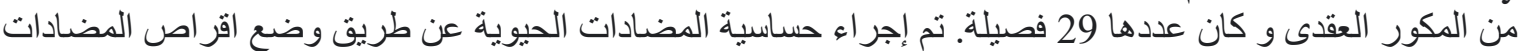

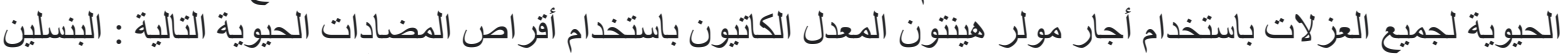

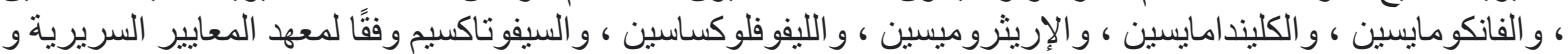
المخبرية (2015).

النتائج: تم تسجيل أعلى مستوى من المقاومة ضد السيفوتاكسيم حيث كانت مقاومة 29/20 (69٪). بينما تم تسجيل الحد الأدنى من المقاومة ضد الليفوفلوكساسين بمعدل مقاومة 27,5٪ (29/8).

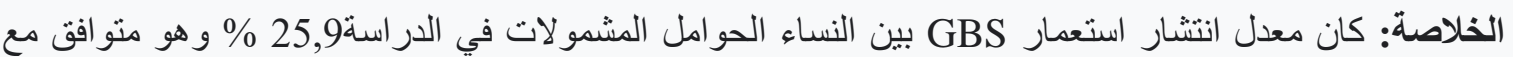

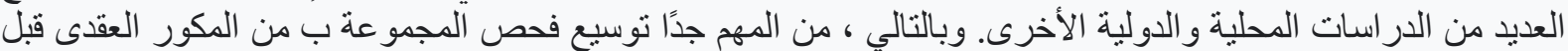

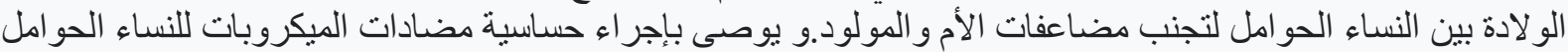

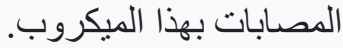

